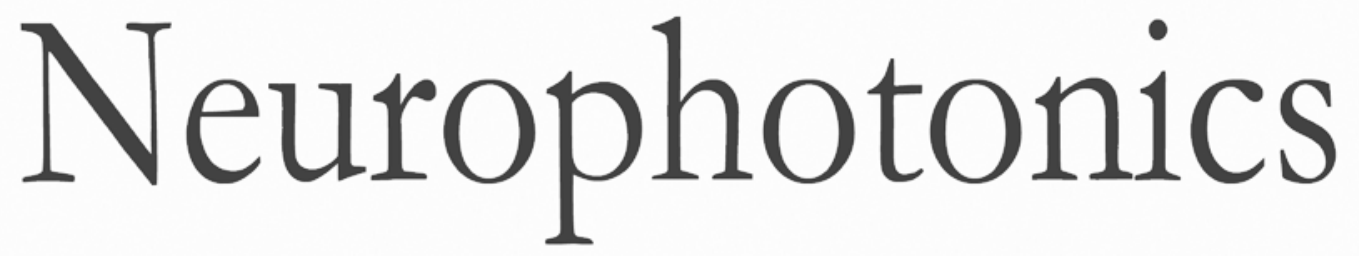

Neurophotonics.SPIEDigitalLibrary.org

\title{
Neurophotonics 2014 List of Reviewers
}

\section{SPIE.}


Neurophotonics would like to sincerely thank the following individuals who served as reviewers in 2014 . The success of our publication hinges on the voluntary contributions of time and energy put forth by these professionals.

Anna Letizia Allegra Maso
Afrouz Anderson
Srdjan Antic
Bradley Baker
Anna Blasi
Pablo Blinder
David Boas
Edward M. Callaway
Marco Canepari
Jean-Yves Chatton
Yu Chen
Radim Chmelik
Robert Cooper
Ippeita Dan
Vincent Ricardo Daria
Hamid Dehghani
Suzie Dufour
Andrew Dunn
Turgut Durduran
Adam Eggebrecht
Amanda Foust
Maria Franceschini
Dan Fu
Amir Gandjbakhche
Xue Han
Fumitaka Homae
Song Hu
Meyer Jackson

Adam Kampff
Satoko Kawauchi
Bjoern Kemper
Bilal Khan
Beop-Min Kim
Kazuo Kitamura
Joel Kralj
Kishore Kuchibhotla
Flavie Lavoie-Cardinal
Terence Leung
Ofer Levi
Sarah Lloyd-Fox
Sylvie Lorthois
Qingming Luo
Tianyi Mao
Jose Martinez Lorenzo
Liset Menendez de la Prida
Inkyu Moon
Gerhard Multhaup
Timothy Murphy
Nozomi Naoi
Theoden Netoff
Vasilis Ntziachristos
Masako Okamoto
Jon Onativia
B. Hyle Park
Luc Pellerin
Katherine Perdue

Marko Popovic
Jessica Ramella-Roman
Mahsa Ranji
Claus-Peter Richter
William Ross
Edward Ruthazer
Sava Sakadzic
Brian Salzberg
Puifai Santisakultarm
James Schummers
Andy Shih
Vivek Srinivasan
Cha-Min Tang
Peifang Tian
Daisuke Tsuzuki
Vassiliy Tsytsarev
Shinji Umeyama
Benjamin Vakoc
Pu Wang
Shouyi Wang
Teresa Wilcox
Arnold Wilkins
Toru Yamada
Jong Ye
Ji Yi
Meryem Yucel
Dejan Zecevic

